\title{
Development of a Fountain Detector for Spectroscopy of Secondary Electrons in SEM
}

Toshihide Agemura ${ }^{1,2}$, Hideo Iwai $^{1}$ and Takashi Sekiguchi ${ }^{1,2}$

1. National Institute for Materials Science, Nano-Electronics Material Unit, Tsukuba, Japan

2. University of Tsukuba, Graduate School of Pure and Applied Sciences, Tsukuba, Japan

Scanning electron microscopes (SEM) are widely used to observe surface structures of various materials on the nanometer scale. To improve the image resolution, several modifications are implemented; the immersion objective lens that strong magnetic fields are immersed onto specimens, the composed objective lens that electrostatic and magnetic fields are superimposed, the retarding voltage that a high voltage is biased onto the specimens, and so on. However, it must be pointed that SEM users cannot estimate the energy, angle and number of electrons emitted from the specimen, because those complicated systems with the electrostatic and magnetic fields change the energy and angle of the emitted electrons.

It is well-known that the emitted electrons are categorized into secondary electrons (SE) whose emitted electron energy is less than $50 \mathrm{eV}$ and back scattered electrons (BSE) whose emitted electron energy is in the range from $50 \mathrm{eV}$ to the landing energy of primary electrons onto the specimens. Recently, energy and angular selective detections of the BSEs became commercially available by adopting simple energy filters and a segmented solid-state detector. However, the energy and angular selective detectors of the SEs are not commercially available unless expensive semi-spherical or cylindrical energy analyzers are mounted, because the energy of the SEs is very low and those SEs are severely suffered from the electrostatic and magnetic fields of the systems. Thus, the SEs are not effectively used to characterize the physical/chemical properties of materials.

To overcome such difficulties, we have developed a detector which can be mounted on an out-lens type SEM, which the SEs do not sense the electrostatic and magnetic fields before the detection [1]. This detector is very simple and composed of planar filter grids above and a micro-channel plate (MCP) below the specimen. Figure 1 shows an appearance of the detector. The SE trajectories become parabolic, which looks like a "fountain" and can be simulated easily as shown in Figure 2 . The angular selective detection is also realized by a sectoring MCP.

However, it has been founded that there are many tertiary electrons (TEs) which increase the noise level of the detector, because the energy of the TEs is the same as that of SEs. The BSEs emitted from the specimen hit the grids and yield significant TEs. In order to fix this problem, we have developed a new detector with spherical filter grids, which the open ratio of the grids is increased. Based on the simulation, the spherical grids increase the SE detection efficiency three times, and the increased open ratio decreases the noise level as a factor of four.

In the presentation, we show the improvement of detector performance and give the instances of SE observation using this detector. 
Reference:

[1] T. Sekiguchi and H. Iwai, Japanese Journal of Applied Physics 54 (2015), p. 088001.

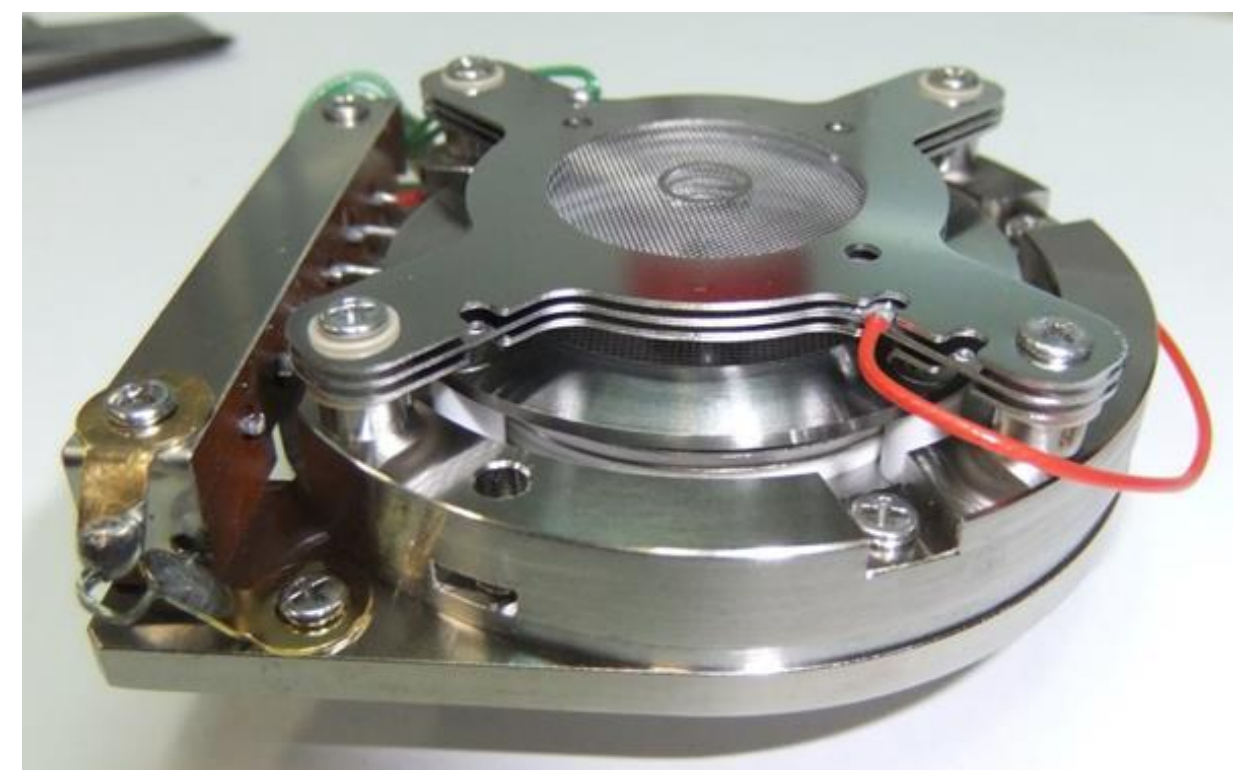

Figure 1. Appearance of the planner type fountain detector.

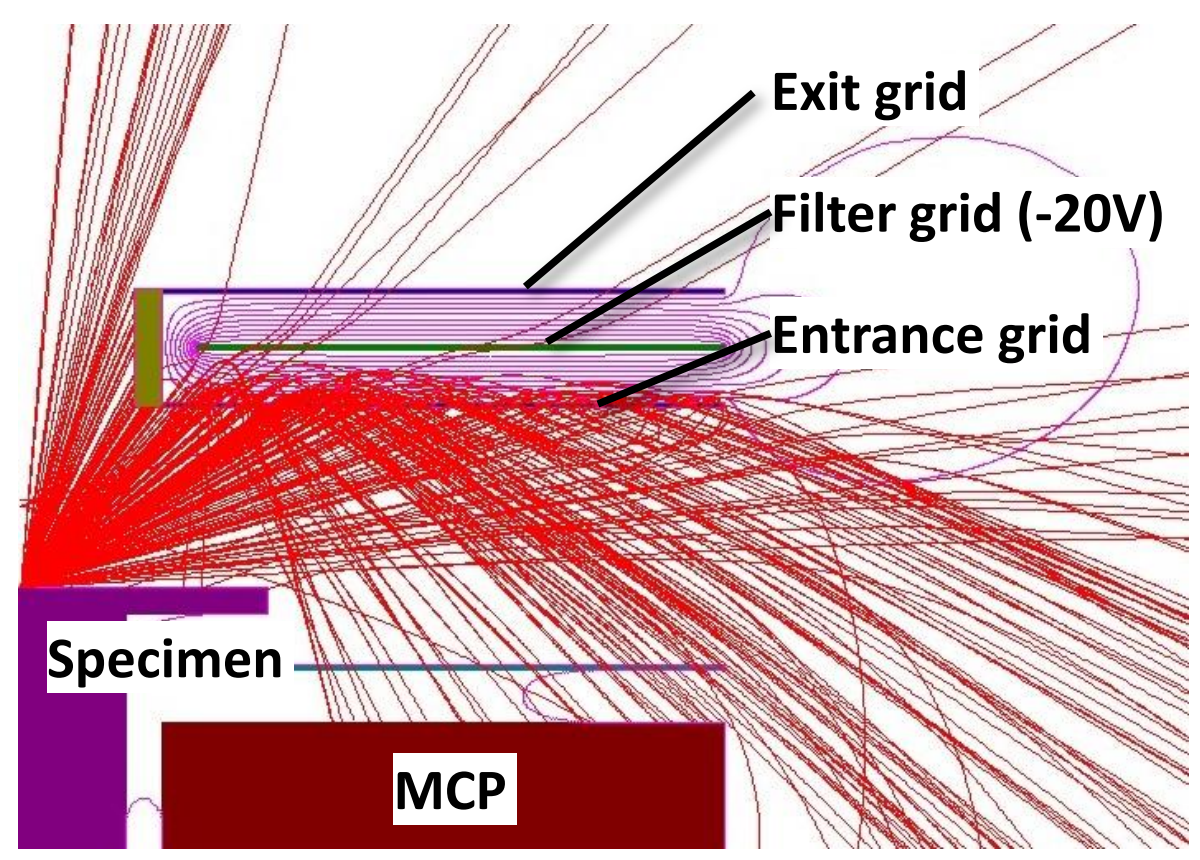

Figure 2. Simulation of the SE trajectories. The red and pink lines represent the SE trajectories and the equi-potential lines respectively. The voltage on the filter grid was $-20 \mathrm{~V}$. The space between two lines indicates the voltage difference of $2 \mathrm{~V}$. 